\title{
POLICY PLATFORMS, CAMPAIGN SPENDING AND VOTER PARTICIPATION
}

\author{
HELIOS HERRERA, DAVID K. LEVINE AND CÉSAR MARTINELLI
}

\begin{abstract}
We model electoral competition between two parties in a winner-take-all election. Parties choose strategically first their platforms and then their campaign spending under aggregate uncertainty about voters' preferences. We use the model to examine why campaign spending in the United States has increased at the same time that politics has become more polarized. We find that the popular explanation - better targeting of campaign spending - is not a likely explanation. While better targeting does lead to greater spending, it leads to less polarization. Instead we argue that the likely explanation is that voters preferences have become more volatile. This will both raise campaign spending and increase polarization. At the same time it is consistent with the observation that voters have become less committed to the two parties.
\end{abstract}

Keywords: Non Voting Paradox, Electoral Platforms, Turnout.

JEL Classification: D72

Date: First Version: February 7th 2004, This Version: 18th July 2005.

We thank the National Science Foundation for financial support.

Herrera: CIE-ITAM, Camino Santa Teresa 930, 10700 Mexico, D.F., MEXICO. Email: helios@itam.mx. Levine: Department of Economics, UCLA, Los Angeles, CA 90095, USA. Email: davidedklevine.com. Martinelli: CIE-ITAM, Camino Santa Teresa 930, 10700 Mexico, D.F., MEXICO. Email: martinel@itam.mx. 


\section{INTRODUCTION}

High turnout in large elections or the "non voter paradox" has been considered a major problem for the modelling of voters as instrumentally rational agents at least since Downs [3]. While voting costs may be low, the probability of affecting the outcome of the election is so tiny in a large election that even very small costs should deter any individual motivated solely by the desire to influence the election result from going to vote. The gametheoretic analysis of Palfrey and Rosenthal [14], built on earlier work by Ledyard [7], confirms Downs's intuition: in large elections, Nash equilibrium voter turnout is extremely low, provided that voters are somewhat uncertain about the preferences or voting inclination of others. This is clearly at odds with regularly observed large turnouts. That is, there must be something more than the simple desire to influence the election result that brings people to the voting booth on election day. In fact, political parties spend considerable money and effort to encourage people to vote. This includes such things as decreasing the direct cost of voting - for example by providing volunteers who drive voters to the polls; decreasing the cost of acquiring information - for example by publicizing attractive aspects of their platforms and candidates and negative aspects of their rivals; increasing the cost of not voting - for example via social sanctions; and by signaling the closeness and importance of the election race. By the same token, we may expect political parties to take into account the expected cost of bring voters to the booth when formulating electoral platforms.

In this paper, we model electoral competition as a two-stage game. In the first stage, two parties (with both an ideological and an office motivation) strategically choose their platforms. In the second stage, parties decide how much to spend on the campaign. Turnout for each party is a function of campaign spending as well as voters' bias in favor of one or the other party. We treat party bias as being independent of parties' platforms and subject to aggregate shocks. We can think of party bias as reflecting voters' concerns for issues that are "symbolic" rather than related to policy decisions. We consider campaign spending as having an impact on turnout via mobilization of voters. We pay special attention to the effectiveness of campaign targeting. If the targeting ability of parties is low, then campaign spending partially misfires, by mobilizing voters in favor of the other party; if the targeting ability of parties is high, each party's spending mobilizes only voters in favor of that party.

We use the model to explore three stylized facts about electoral politics in the United States.

- The two parties have become increasingly polarized.

- Campaign spending has increased substantially. 
- Voters have become less committed to the two parties.

Poole and Rosenthal [15] and McCarty, Poole and Rosenthal [10] provide some evidence on polarization, based on the average distance between Democratic and Republican members of Congress on a liberal-conservative scale. They find that polarization has been sharply increasing since around 1980, after a long period of decline started around 1900.

With respect to campaign spending, and more generally the campaign effort of political parties and allied interest groups, an interesting indirect source is the percentage of respondents in public opinion studies contacted by political parties in elections. National Election Studies [12] (Tables 6C.1a, 6C.1b and 6C.1c), provides evidence of a sharp increase in the percentage of respondents contacted by either party since 1990 .

Finally, with respect to the commitment of voters to the two parties, party affiliation has fallen enormously since 1960. According to some observers, the fraction of voters who register as neither Democrat nor Republican has gone from 1.6 in 1960 to 21.7 in 2004 (see [2], p. 11). The party identification data from the National Election Studies [12] (Tables 2A.1, 2A.2 and 2A.3) is consistent with this view. The percentage of voters who declare themselves as independent or leaning independent has gone from 25 in 1960 to 37 in 2002.

We consider two possible explanations of these facts. First, commentators have also suggested that the reason for both the increased polarization and campaign spending is because skilled political operatives using sophisticated statistical tools, and purchasing advertising in local markets are better able to target particular voters (see e.g. [17]). However, in our model improved targeting may indeed lead to an increase in campaign spending but it also leads to a reduction in polarization. The reason for the reduction in polarization is that, in deciding their policy platforms in the first stage of the game, parties anticipate an increase in campaign costs in the second stage as a result of better targeting. Polarized platforms become too costly.

The second explanation - and our favored one - is that voters preferences have become more volatile. By increased volatility, we mean larger aggregate shocks to party bias. We show in our model that increase in volatility leads to both an increase in campaign spending and an increase in polarization. Moreover, it also provides an explanation of the decreasing commitment of voters to the two parties: a voter who is relatively near the middle will have little reason to affiliate with a particular party since it will be difficult for her to predict which party she would prefer.

The effect of volatility on polarization is very intuitive. Greater volatility means that the results of elections are less certain. Consequently, the 
parties have less reason to please the center voters, and are free to move towards their own extreme preferences. The effect of volatility on campaign spending is less intuitive. We can decompose it in two effects. First, holding fixed the party positions, increasing volatility unambiguously lowers spending. However, increasing volatility also increases polarization in the first stage of the game. That means that in the second stage game, the stakes are higher - it is better to win and worse to lose. That increases the marginal benefit of spending. So there are two offsetting effects, and the comparative static theorem shows that the increased spending dominates once electoral uncertainty is big enough.

Recently, Dekel, Jackson and Wolinsky [4] have devoted some attention to the issue of buying votes using different procedures. In their setup, campaign expenditure is more effective and less is spent if the parties can buy binding commitments to vote ("up front vote buying"). In comparison, in our setup voters cannot make binding commitments with parties, but parties have an (imperfect) ability to target their spending to favorable voters.

\section{THE MODEL}

We model a winner-take-all election between two parties, $\mathbf{D}$ and $\mathbf{R}$. The election takes place in two stages. In the first stage, the two parties simultaneously choose binding policy platforms $d$ and $1-r$, which are elements of the policy space $[0,1]$. In the second stage, observing the policy platforms of the other party, they simultaneously choose their campaign efforts $D$ and $R$, which are elements of the effort space $[0,1]$.

Each party has an "office motivation" for winning the election, which we represent as an amount $G \geq 0$ for winning the election. Each party also cares about the policy $p$ implemented by the winning party, which we represented by $g(p), g(1-p)$ for $\mathbf{D}$ and $\mathbf{R}$ respectively. Finally, the campaign effort of

a party $E=D, R$ has a cost $c(E)$. Overall, party $\mathbf{D}$ and party $\mathbf{R}$ 's payoffs are

$$
V^{\mathrm{D}}=\left\{\begin{array}{ll}
G+g(d)-c(D) & \text { if party } \mathbf{D} \text { wins } \\
g(1-r)-c(D) & \text { if party } \mathbf{R} \text { wins }
\end{array},\right.
$$

and

$$
V^{\mathrm{R}}=\left\{\begin{array}{ll}
G+g(r)-c(R) & \text { if party } \mathbf{R} \text { wins } \\
g(1-d)-c(R) & \text { if party } \mathbf{D} \text { wins }
\end{array} .\right.
$$

We assume that the functions $g, c$ are decreasing and increasing respectively, and have the isoelastic forms

$$
\begin{gathered}
g(p)=\left\{\begin{array}{cc}
(1 / 2-p)^{\beta} & \text { if } p \in[0,1 / 2] \\
-(p-1 / 2)^{\beta} & \text { if } p \in(1 / 2,1]
\end{array}\right. \\
c(E)=\delta E^{1+\gamma}
\end{gathered}
$$


for $\beta \in(0,1]$ and $\delta>0$ and $\gamma \geq 0$. Note that in this formulation, parties prefer more extreme positions.

The policy motivation function $g$ is therefore strictly decreasing on $[0,1]$ and concave and continuously differentiable on $[0,1 / 2)$. This means that party $\mathbf{D}$ and party $\mathbf{R}$ 's ideal points in the policy space are, respectively, 0 and 1. Observe that $\beta=1$ represents the familiar Euclidean policy preferences, while $\beta$ near zero represents parties that are (almost) purely officemotivated. Notice also that $g(p)=-g(1-p)$; this means that the sum of the payoffs of the two parties is constant regardless of who wins the election.

The outcome of the election is determined by the voters, of whom there is a continuum uniformly distributed on the unit interval and indexed by $v \in[0,1]$. Voters' preferences are determined jointly by party positions and by "party identification," as modelled by Lindbeck and Weibull $[8,9]$ and others. As described below, voters will not necessarily turn out to vote, so the determinant of the election is the fraction that favor either party and turn out to vote.

Policy preferences of voters are Euclidean with their ideal point determined by their index $v$. In addition to their policy preferences, voters have an idiosyncratic party bias $b_{v}$ in favor of $\mathbf{D}$ and an aggregate party bias $b$, also in favor of $\mathbf{D}$. So voter $v$ will favor party $\mathbf{D}$ if

$$
-|v-d|+b_{v}+b>-|v-(1-r)|
$$

and will favor party $\mathbf{R}$ if the inequality is reversed.

We assume that $b$ is randomly distributed according to the distribution $F$ with twice continuously differentiable density $f$ and support $[-\alpha, \alpha]$, and that $b_{v}$ is uniformly distributed with support $[-1-\alpha, 1+\alpha]$, where $\alpha \geq 1 / 4$. The realization of $b$ is is not known to parties until after they propose their policy position and carry out their campaign spending. Notice that $\alpha$ is a measure of the volatility of voter preferences.

Voters do not necessarily show up to vote for the party they favor. Rather, the numbers that show up are determined by the effort made by each party to turn out the vote. A fraction $t D+(1-t) R$ of voters favoring party $\mathbf{D}$ and a fraction $t R+(1-t) D$ of voters favoring party $\mathbf{R}$ show up to vote for the parties they favor, while the other voters abstain. The parameter $t \in(1 / 2,1]$ represents the accuracy of campaign targeting. If $t=1$, then $D, R$ represent how many (what fraction) of voters each party chooses to turn out; as we have assumed, the cost of increasing turnout $c$ exhibits diminishing returns to scale, it is increasingly difficult to persuade voters favoring either party to show up to vote. If $t<1$, some of the campaign spending of each party misfires, by mobilizing voters in favor of the other party. We can think of campaign effort as reducing the cost of showing up for voters or as attaching 
some "symbolic value" to the act of voting. 1 The election is won by the party with more votes.

To guarantee concavity of the objective function of parties with respect to campaign spending, we assume that the density $f$ does not decrease too rapidly in the sense that

$$
-f^{\prime}(z) / f(z) \leq \frac{1}{(1+\alpha)(2 t-1)}
$$

for any $z$ in the support of $F$. When $F$ is uniform this is always satisfied. To avoid dealing with corner cases, we assume that the marginal cost of effort $\delta$ is sufficiently high that neither party is inclined to get all the favorable voters to vote

$$
(1+\gamma) \delta \geq(1+\alpha)(2 t-1)\left(2^{-\beta}+G / 2\right) \sup _{z} f(z)
$$

We also assume that $\gamma$, the curvature of the cost of effort, and $\beta$, the curvature of the policy reward are both sufficiently large that

$$
\frac{1}{\frac{2\left(2(1 / 2)^{\beta}+G\right)}{\beta(1 / 2)^{\beta-1}}+\frac{(1+\alpha)(2 t-1)}{1+\gamma}}<\frac{1}{2 \alpha}<\frac{1+\gamma}{(1+\alpha)(2 t-1)} .
$$

\section{EQUiLIBRIUM}

From the model, we can work out the probability that each party wins and voter turnout as a function of the policy platforms and campaign spending.

Theorem 3.1. The fraction of voters favoring party $\boldsymbol{D}$ is

$$
1 / 2+\left(b+d-d^{2}-r+r^{2}\right) /(2+2 \alpha) .
$$

with the remainder favoring party $\boldsymbol{R}$. The probability that party $\boldsymbol{D}$ wins is

$$
1-F\left(\left(-d+d^{2}+r-r^{2}\right)-(1+\alpha)(2 t-1)(D-R) /(D+R)\right) .
$$

Aggregate voter turnout is

$$
(D+R) / 2+(D-R)(2 t-1)\left(b+d-d^{2}-r+r^{2}\right) /(2+2 \alpha) .
$$

All proofs may be found in the Appendix. In the expression for the probability of $\mathbf{D}$ winning, the first term in the argument of the distribution function

$$
-d+d^{2}+r-r^{2}
$$

\footnotetext{
${ }^{1}$ Note that the cost depends upon the fraction rather than the absolute number; so if a party has very few favorable voters, it is just as costly to turn out half of them as if the party has a lot of favorable voters. This assumption may make more sense when dealing with national parties with geographically dispersed constituencies.
} 
represents the influence of policy platforms, while the second term

$$
-(1+\alpha)(2 t-1)(D-R) /(D+R)
$$

represents the influence of campaign spending on the election outcome. (If $D=R=0$, we assume that each party has a positive probability of winning the election.) Campaign spending has potentially at least three roles: (1) Move party sympathizers to effectively vote; (2) Persuade undecided voters or voters leaning to the other party of the merits of one party's policies; (3) Dissuade sympathizers of the other party to vote. We have focused on the "mobilization" aspect of campaign spending rather than on the "persuasion" or "vote suppression" aspects.

Given the probabilities of winning, we can work out the second stage equilibrium campaign spending given policy platforms.

Theorem 3.2. If $g(d)+g(r)+G<0$, then the unique second stage Nash choice of campaign spending is $D=R=0$. If $g(d)+g(r)+G \geq 0$ both parties spend the same amount $E^{*}$, determined implicitly by

$$
\begin{aligned}
2 E^{*} c^{\prime}\left(E^{*}\right) & = \\
& (1+\alpha)(2 t-1) f\left(-d+d^{2}+r-r^{2}\right)(g(d)+g(r)+G) .
\end{aligned}
$$

\section{THE UNIFORM/SyMMETRIC CASE}

As we have seen, campaign spending by the two parties is equal even if $F$ is asymmetric - provided only that it does not decrease too rapidly. We will now focus on the symmetric case in which $F$ is actually uniform on $[-\alpha, \alpha]$. In this case we can solve to find the first stage equilibrium, which is unique and symmetric. For notational simplicity, we let $h=1 / 2 \alpha$ denote the height of the density function.

Theorem 4.1. If $F$ is uniform on $[-\alpha, \alpha]$, there is a unique equilibrium, it is symmetric, and each party chooses the platform given implicitly by

$$
g^{\prime}\left(p^{*}\right)\left(\frac{1}{2}-\frac{1+\alpha}{2+2 \gamma}(2 t-1) h\right)+\left(2 g\left(p^{*}\right)+G\right) h\left(1-2 p^{*}\right)=0 .
$$

The solution to this equation has $0<p^{*}<1 / 2$.

\section{Comparative Statics}

In this section we perform some comparative static exercises on the uniform symmetric case described in the previous section.

Theorem 5.1. If $F$ is uniform on $[-\alpha, \alpha]$, the equilibrium policy position $p^{*}$ is independent of $\delta$, increasing in $G, t$ and decreasing in $\alpha, \gamma$. Equilibrium 
campaign spending $E^{*}$ decreases with $\delta$ and increases with $G$. Moreover, if $G=0$,

$$
\frac{\partial E^{*}}{\partial t} \gtreqless 0 \Longleftrightarrow t \lesseqgtr 1 / 2+\frac{2 \alpha(1+\gamma)}{(2+\beta)(1+\alpha)}
$$

and

$$
\frac{\partial E^{*}}{\partial \alpha} \gtreqless 0 \Longleftrightarrow \alpha^{2}-(2 / \beta-1) \alpha+\frac{2 / \beta}{\frac{1+\gamma}{t-1 / 2}-1} \gtreqless 0 .
$$

This result provides unambiguous predictions with respect to the effects of the parameter of the model on polarization $\left(1 / 2-p^{*}\right)$. In particular, an increase in the accuracy of campaign targeting reduces polarization, and an increase in electoral uncertainty increases polarization. The effect of electoral uncertainty on polarization is quite intuitive. Indeed, previous literature at least since the work of Wittman [18] and Calvert [1] has shown that increased electoral uncertainty can dampen the incentive of policymotivated parties to moderate their electoral platforms. Per contra, our result on the effect of targeting accuracy on polarization is novel. Intuitively, if parties' platforms were to stay constant, an increase in targeting accuracy would lead to an increase in campaign costs in the second stage of the model. This reduces the incentive for parties to diverge in the first stage of the model.

The effects of the parameters of the model on campaign spending (and thus on turnout) are not clear cut. This is because parties set their policy choices anticipating the campaign stage of the electoral game. Thus, the direct effect of the underlying parameters on campaign spending may be undone by indirect effects through policy choices. For instance, from equation 7.6, we can see that holding policy choices constant, an increase in the accuracy of campaign spending increases spending. However, increased accuracy also reduces polarization, thereby reducing the incentive to invest in campaigning. Similarly, an increase in electoral uncertainty has a negative direct effect on campaign spending but a positive indirect effect.

From equation 7.6, we expect indirect effects to be particularly strong if there is no office motivation $(G=0)$ or office motivation is relatively small. Theorem 5.1 provides some comparative statics results with respect to campaign spending for the case $G=0$. We obtain that the net effect of greater accuracy in targeting over campaign spending is positive if campaign spending is not very accurate to begin with $(t$ near $1 / 2)$, or if electoral uncertainty is large and the elasticity of campaign costs $1+\gamma$ is large. Similarly, manipulating the quadratic condition for $\partial E^{*} / \partial \alpha$ in Theorem 5.1, we get that the net effect of larger electoral uncertainty over campaign spending is negative 
if and only if

$$
\frac{1}{\beta}-\frac{1}{2}-\sqrt{\left(\frac{1}{\beta}-\frac{1}{2}\right)^{2}-\frac{2 \beta}{\frac{1+\gamma}{t-1 / 2}-1}}<\alpha<\frac{1}{\beta}-\frac{1}{2}+\sqrt{\left(\frac{1}{\beta}-\frac{1}{2}\right)^{2}-\frac{2 \beta}{\frac{1+\gamma}{t-1 / 2}-1}}
$$

and the term under square root is positive; that is

$$
t<\frac{1}{2}+\frac{1+\gamma}{1+\frac{\beta}{(1 / \beta-1 / 2)^{2}}} .
$$

Note that the net effect of greater electoral uncertainty over campaign spending is positive if electoral uncertainty is large, or if campaign spending is accurate and $\gamma$ is small.

For instance, under the usual (but extreme) assumptions of Euclidean preferences $(\beta=1)$ and linear costs $(\gamma=0)$, if $G=0$ then the net effect of greater accuracy in targeting over campaign spending is positive if $t<$ $1 / 2+2 \alpha /(3+3 \alpha)$, and the net effect of greater electoral uncertainty over campaign spending is positive if $t>2 / 3$.

\section{CONCLUSION}

Our goal has been to understand why campaign spending has increased at the same time that politics has become more polarized. Theorem 5.1 shows us that an improvement in targeting alone is not enough to explain both trends. Improving targeting $t$ may lead to an increase in campaign spending (particularly if $G$ is not small) but it also leads to a reduction in polarization. That is, with better targeting parties compete more both by spending more and increasing attention given to the the median voter, that is by being less polarizing.

On the other hand, an increase in $\alpha$ the volatility of voter preferences does lead both to an increase in campaign spending (at least if $\alpha$ is big enough), and also to an increase in polarization. As we noted, it is also potentially an explanation for the increasing lack of party affiliation. We treat office motivation $G$ as exogenous; but it may very well be that as fewer voters have a party affiliation, parties fall into the hands of extremists, which are more motivated by policy considerations than by holding office - this reinforces the effect that is in the model.

In the model we ignore the possibility of campaign contribution limits being actually binding. ${ }^{2}$ In fact, the model suggests a way to limit he waste

\footnotetext{
${ }^{2}$ For instance, referring to the 2004 Congressional election, Jacobson [6] states that the flow of campaign funds has been much less determined by the campaign reform act of 2002 than by the usual strategic considerations.
} 
associated with costly campaigning in the context of polarized politics. Taxing campaign contributions and money spent by advocacy groups would be equivalent in our model to raising $\delta$. Note that party positions are independent of $\delta$ (Theorem 5.1). Moreover, using equation 7.6, it is easy to see that campaign $\operatorname{costs} c(E)$ remain constant if $\delta$ changes. This is because changes in $\delta$ are completely offset in equilibrium by changes in campaign effort. Thus, taxing campaign contributions simply takes resources away from a wasteful activity. 


\section{APPENDIX}

Theorem. 3.1 The fraction of voters favoring party $\boldsymbol{D}$ is

$$
1 / 2+\left(b+d-d^{2}-r+r^{2}\right) /(2+2 \alpha) .
$$

with the remainder favoring party $\boldsymbol{R}$. The probability that party $\boldsymbol{D}$ wins is

$$
1-F\left(\left(-d+d^{2}+r-r^{2}\right)-(1+\alpha)(2 t-1)(D-R) /(D+R)\right) .
$$

Aggregate voter turnout is

$$
(D+R) / 2+(D-R)(2 t-1)\left(b+d-d^{2}-r+r^{2}\right) /(2+2 \alpha) .
$$

Proof. From the preceding assumptions, if $d \leq 1-r$,

$\operatorname{Pr}\{$ voter $v$ favors party $\mathbf{D}\}=\left\{\begin{array}{ll}\frac{1}{2}+\frac{1-r-d+b}{2+2 \alpha} & \text { if } 0 \leq v \leq d \\ \frac{1}{2}+\frac{1-r+d+b-2 v}{2+2 \alpha} & \text { if } d \leq v \leq 1-r . \\ \frac{1}{2}+\frac{r-1+d+b}{2+2 \alpha} & \text { if } 1-r \leq v \leq 1\end{array}\right.$.

Integrating this over voters $v$ we get the overall fraction favoring party $\mathbf{D}$

$$
\begin{gathered}
d\left(\frac{1}{2}+\frac{1-r-d+b}{2+2 \alpha}\right)+(1-r-d)\left(\frac{1}{2}+\frac{1-r+d+b}{2+2 \alpha}\right) \\
+r\left(\frac{1}{2}+\frac{r-1+d+b}{2+2 \alpha}\right)+\int_{v=d}^{v=1-r} \frac{-2 v}{2+2 \alpha} d v \\
=\frac{1}{2}+\frac{\left(b+(1-r)^{2}+(r-1) r+d(1-d)-d^{2}\right)}{2+2 \alpha} \\
-\frac{\left((1-r)^{2}-d^{2}\right)}{2+2 \alpha} \\
=\frac{1}{2}+\frac{\left(b+d-d^{2}-r+r^{2}\right)}{2+2 \alpha} .
\end{gathered}
$$

Similar calculations show that the same expression applies in case $d>1-r$. This in turn implies that, the probability that party $\mathbf{D}$ wins is equal to the probability that

$$
\begin{aligned}
D\left(t-\frac{1}{2}+\frac{b+d-d^{2}-r+r^{2}}{2+2 \alpha}\right) & \\
& >R\left(t-\frac{1}{2}-\frac{b+d-d^{2}-r+r^{2}}{2+2 \alpha}\right)
\end{aligned}
$$

or

$$
b>\frac{2+2 \alpha}{D+R}\left(-(D-R)\left(t-\frac{1}{2}\right)-(D+R) \frac{d-d^{2}-r+r^{2}}{2+2 \alpha}\right) .
$$

This implies the overall probability that $\mathbf{D}$ wins is the expression above. 
Aggregate voter turnout is given by adding the two sides of 7.1 gives the aggregate voter turnout

$D\left(\frac{1}{2}+(2 t-1) \frac{b+d-d^{2}-r+r^{2}}{2+2 \alpha}\right)+R\left(\frac{1}{2}-(2 t-1) \frac{b+d-d^{2}-r+r^{2}}{2+2 \alpha}\right)$

which simplifies to the expression above.

Theorem. 3.2 If $g(d)+g(r)+G<0$, then the unique second stage Nash choice of campaign spending is $D=R=0$. If $g(d)+g(r)+G \geq 0$ both parties spend the same amount $E^{*}$, determined implicitly by

(7.2) $2 E^{*} c^{\prime}\left(E^{*}\right)=$

$$
(1+\alpha)(2 t-1) f\left(-d+d^{2}+r-r^{2}\right)(g(d)+g(r)+G) .
$$

Proof. Suppose that parties have chosen their policy platforms in the first stage of the game and consider their choice of campaign spending in the second stage. Let the parties be $i=\mathbf{D}, \mathbf{R}$, and let $p_{i}=d, r$ and $E_{i}=D, R$. Let $F_{i}$ denote $F$ if $i=\mathbf{D}$ and $1-F$ if $i=\mathbf{R}$. The objective function of party $i$ is

$$
\begin{gathered}
\left(1-F_{i}\left(-d+d^{2}+r-r^{2}-(1+\alpha)(2 t-1)(D-R) /(D+R)\right)\right)\left(g\left(p_{i}\right)+G\right) \\
+F_{i}\left(-d+d^{2}+r-r^{2}-(1+\alpha)(2 t-1)(D-R) /(D+R)\right) g\left(1-p_{-i}\right) \\
-c\left(E_{i}\right)
\end{gathered}
$$

or equivalently,

$$
\begin{aligned}
-F_{i}\left(-d+d^{2}+r-r^{2}-(1+\alpha)(2 t-1) \frac{D-R}{D+R}\right) & (g(d)+g(r)+G) \\
& -c\left(E_{i}\right)+g\left(p_{i}\right)+G .
\end{aligned}
$$

It is easy to check that if $g(d)+g(r)+G \leq 0$, then the unique Nash choice of campaign spending is $D=R=0$, as the payoff of winning the election will not be positive. Now consider the case in which $g(d)+g(r)+G>0$ (as will hold in the subgame perfect equilibrium analyzed in the next section). It is easy to show that there is no Nash equilibrium in which either one or the two parties do not spend any positive amount. The following first order condition must hold for $i=\mathbf{D}, \mathbf{R}$ in any Nash equilibrium if both parties spend positive amounts:

$$
\begin{aligned}
c^{\prime}\left(E_{i}\right)=f(-d & \left.+d^{2}+r-r^{2}-(1+\alpha)(2 t-1)(D-R) /(D+R)\right) \\
& \times(g(d)+g(r)+G)(1+\alpha)(2 t-1)\left(2 E_{-i}\right)(D+R)^{-2} .
\end{aligned}
$$

(Corner solutions in which either party chooses effort level 1 are ruled out by our lower bound on $c^{\prime}(1)$.) 
From the previous equation,

$$
\begin{array}{r}
c^{\prime}\left(E_{i}\right) / E_{-i}=f\left(-d+d^{2}+r-r^{2}-(1+\alpha)(2 t-1)(D-R) /(D+R)\right) \\
\times(g(d)+g(r)+G)(1+\alpha)(4 t-2)(D+R)^{-2} .
\end{array}
$$

which implies $c^{\prime}(D) / R=c^{\prime}(R) / D$ or $D c^{\prime}(D)=R c^{\prime}(R)$. Since $x c^{\prime}(x)$ is strictly increasing in $x$, it follows that $D=R$. Using $D=R=E^{*}$ and simplifying the previous equation we get 7.2.

We want to check that the second order condition holds for both parties so that in fact we have found the (unique) Nash equilibrium choice of campaign spending for any given pair $d, r$. The second derivative of the objective function of party $\mathbf{D}$ is

$$
\begin{aligned}
-f(z)(1+\alpha)(2 t & -1)(k)(4 R)(D+R)^{-3} \\
& +f^{\prime}(z)(1+\alpha)^{2}(2 t-1)^{2}(k)\left(4 R^{2}\right)(D+R)^{-4}-c^{\prime \prime}(D)
\end{aligned}
$$

where

$$
z=-d+d^{2}+r-r^{2}-(1+\alpha)(2 t-1)(D-R) /(D+R)
$$

and

$$
k=g(d)+g(r)+G .
$$

Rewriting the second derivative,

$-4(1+\alpha) k R(D+R)^{-3}\left(f(z)-f^{\prime}(z)(1+\alpha)(2 t-1)(R)(D+R)^{-1}\right)-c^{\prime \prime}(D)$.

Since $-f^{\prime}(z) / f(z) \leq 1 /[(1+\alpha)(2 t-1)]$ by assumption, we get that

$$
f(z)-f^{\prime}(z)(1+\alpha)(2 t-1)(R)(D+R)^{-1}>0
$$

for any $d, r, D, R$. Thus, the second derivative of the objective function of party $\mathbf{D}$ is negative, i.e. the objective function of party $\mathbf{D}$ is concave. A similar calculation shows that the objective function of party $\mathbf{R}$ is also concave.

We now prove a series of Lemmas leading up to the proof of Theorem 4.1.

Lemma 7.1. Assume $F$ is uniform. Given any $p_{-i}$, party $i$ 's best response policy choice is such that $g\left(p_{i}\right)+g\left(p_{-i}\right)+G \geq 0$.

Proof. We focus on the problem solved by party $\mathbf{D}$. The problem solved by party $\mathbf{R}$ is entirely symmetric.

We claim first that, given any policy choice $r$ by party $\mathbf{R}$, the best response $d^{*}$ by party $\mathbf{D}$ cannot be such that $g\left(d^{*}\right)+g(r)+G<0$ and $d^{*} \geq 1 / 2$. To see this, recall that, from 3.2, if $g(d)+g(r)+G<0$ then the unique second 
stage Nash choice of campaign spending is $D=R=0$. Thus, the objective function of party $\mathbf{D}$ can be written as

$$
-F\left(-d+d^{2}+r-r^{2}\right)(g(d)+g(r)+G)+g(d)+G
$$

over the interval $\{d: g(d)<-g(r)-G\}$. The derivative of the objective function with respect to $d$ is

$$
\left(1-F\left(-d+d^{2}+r-r^{2}\right)\right) g^{\prime}(d)+\frac{2 d-1}{2 \alpha}(g(d)+g(r)+G) .
$$

Since $g(d)+g(r)+G<0$ (by assumption) and $g^{\prime}(d)<0$, the derivative of the objective function is strictly negative for any $d \geq 1 / 2$.

Next, we claim that, given any policy choice $r$ by party $\mathbf{R}$, the best response $d^{*}$ by party $\mathbf{D}$ cannot be such that $g\left(d^{*}\right)+g(r)+G<0$ and $d^{*}<1 / 2$. From the argument in the previous paragraph, the derivative of the objective function with respect to $d$ is nonnegative if and only if

$$
\left(1-F\left(-d+d^{2}+r-r^{2}\right)\right) g^{\prime}(d)+\frac{2 d-1}{2 \alpha}(g(d)+g(r)+G) \geq 0
$$

or equivalently, for $g(d)+g(r)+G<0$ and $d<1 / 2$,

$$
\begin{aligned}
-\beta(1 / 2-d)^{\beta-1}\left(1 / 2+\left(d-d^{2}\right.\right. & \left.\left.-r+r^{2}\right) /(2 \alpha)\right) \\
& +\frac{2 d-1}{2 \alpha}\left((1 / 2-d)^{\beta}+g(r)+G\right) \geq 0 .
\end{aligned}
$$

Note that $g(d)+g(r)+G<0$ implies $r+d>1$, which in turn implies $d-d^{2}-r+r^{2}>0$. Thus, a necessary condition for the derivative of the objective function to be nonnegative is

$$
-\frac{1 / 2-d}{4 \alpha}\left((1 / 2-d)^{\beta}+g(r)\right)>\beta(1 / 2-d)^{\beta-1}(1 / 2) .
$$

Using $g(r) \geq-(1 / 2)^{\beta}$, a necessary condition is

$$
-\frac{1 / 2-d}{4 \alpha}\left((1 / 2-d)^{\beta}-(1 / 2)^{\beta}\right)>\beta(1 / 2-d)^{\beta-1}(1 / 2)
$$

or equivalently,

$$
(1 / 2)^{\beta}>\beta(1 / 2-d)^{\beta-2}(2 \alpha)+(1 / 2-d)^{\beta} .
$$

This condition is not satisfied for any $0 \leq d \leq 1 / 2$, given our parameter constraints $\alpha \geq 1 / 4$ and $0<\beta \leq 1$.

Lemma 7.2. Assume $F$ is uniform. Given any $p_{-i} \leq 1 / 2$, party $i$ 's best response policy choice is such that $p_{i}<1 / 2$. 
Proof. We focus on the problem solved by party $\mathbf{D}$. The problem solved by party $\mathbf{R}$ is entirely symmetric.

Using the previous lemma, we have that, given any policy choice $r$ by party $\mathbf{R}$, the best response $d^{*}$ by party $\mathbf{D}$ cannot be such that $g\left(d^{*}\right)+g(r)+$ $G<0$. Recall that, from Theorem 3.2, if $g(d)+g(r)+G \geq 0$ then the unique second stage Nash choice of campaign spending is given by $E$ as defined in equation 7.2. Using the assumption that the cost function is isoelastic, we have

$$
E c^{\prime}(E)=(1+\gamma) \delta E^{1+\gamma}
$$

Substituting in equation 7.2 , we get

$$
2(1+\gamma) \delta E^{1+\gamma}=(1+\alpha)(2 t-1) h(g(d)+g(r)+G),
$$

or equivalently

$$
2(1+\gamma) c(E)=(1+\alpha)(2 t-1) h(g(d)+g(r)+G)
$$

or

$$
c(E)=\frac{1+\alpha}{2+2 \gamma}(2 t-1) h(g(d)+g(r)+G) .
$$

Recall that the objective function of party $\mathbf{D}$ can be written as

$$
\begin{aligned}
-F_{i}\left(-d+d^{2}+r-r^{2}-(1+\alpha)(2 t-1) \frac{D-R}{D+R}\right) & (g(d)+g(r)+G) \\
& -c\left(E_{i}\right)+g\left(p_{i}\right)+G .
\end{aligned}
$$

Using $D=R=E$ and the previous expression for $c(E)$, we get that the objective function of party $\mathbf{D}$ in the first stage of the game, anticipating correctly the campaign spending choices of both parties, is

$$
\begin{aligned}
-F\left(-d+d^{2}+r-r^{2}\right) & (g(d)+g(r)+G) \\
& -\frac{1+\alpha}{2+2 \gamma}(2 t-1) h(g(d)+g(r)+G)+g(d)+G
\end{aligned}
$$

or equivalently

$$
\begin{array}{r}
(g(d)+g(r)+G)\left(1-F\left(-d+d^{2}+r-r^{2}\right)-\frac{1+\alpha}{2+2 \gamma}(2 t-1) h\right) \\
-g(r)
\end{array}
$$


as long as $g(d)+g(r)+G \geq 0$. The derivative of this objective function with respect to $d$ is

$$
\begin{array}{r}
g^{\prime}(d)\left(1-F\left(-d+d^{2}+r-r^{2}\right)-\frac{1+\alpha}{2+2 \gamma}(2 t-1) h\right) \\
-\frac{2 d-1}{2 \alpha}(g(d)+g(r)+G) .
\end{array}
$$

Now, suppose that, given some policy choice $r \leq 1 / 2$ by party $\mathbf{R}$, the best response $d^{*}$ by party $\mathbf{D}$ is such that $g\left(d^{*}\right)+g(r)+G \geq 0$ and $d^{*}>$ $1 / 2$. Using equation 7.4 , the derivative of the objective function at $d^{*}$ is nonnegative only if

$$
1-F\left(-d^{*}+\left(d^{*}\right)^{2}+r-r^{2}\right)-\frac{1+\alpha}{2+2 \gamma}(2 t-1) h<0 .
$$

Using equation 7.3, the objective function of party $\mathbf{D}$ evaluated at $d^{*}$ is

$$
\begin{array}{r}
\left(g\left(d^{*}\right)+g(r)+G\right)\left(1-F\left(-d^{*}+\left(d^{*}\right)^{2}+r-r^{2}\right)-\frac{1+\alpha}{2+2 \gamma}(2 t-1) h\right) \\
-g(r) .
\end{array}
$$

Note that the first term in this expression is not positive, since $g\left(d^{*}\right)+g(r)+$ $G \geq 0$ and $1-F\left(-d^{*}+\left(d^{*}\right)^{2}+r-r^{2}\right)-\frac{1+\alpha}{2+2 \gamma}(2 t-1) h<0$. Thus, Party $\mathbf{D}$ is better off deviating to $d=r$, because $2 g(r)+G>0$ and $1 / 2-\frac{1+\alpha}{2+2 \gamma}(2 t-$ 1) $h>0$ (using assumption 2.1).

Lemma 7.3. Assume $F$ is uniform. Given any $p_{-i} \leq 1 / 2$, party $i$ 's payoff is strictly concave in its own policy choice in the interval $[0,1 / 2]$.

Proof. We focus on the problem solved by party $\mathbf{D}$. The problem solved by party $\mathbf{R}$ is entirely symmetric.

Suppose that $r \in[0,1 / 2]$, and consider the problem of party D. For $d \leq$ $1 / 2$, we have $g(d)+g(r)+G \geq 0$. Thus, for $d \leq 1 / 2$, the second derivative of the objective function, as given by 7.3 , is

$$
\begin{aligned}
g^{\prime \prime}(d)\left(1-F\left(-d+d^{2}+r-\right.\right. & \left.\left.r^{2}\right)-\frac{(1+\alpha)(2 t-1)}{2+2 \gamma} h\right) \\
& +2 g^{\prime}(d) h(1-2 d)-2(g(d)+g(r)+G) h .
\end{aligned}
$$

Thus, to prove concavity it is enough to show that $g^{\prime \prime}(d)\left(1-F\left(-d+d^{2}+r-r^{2}\right)-\frac{(1+\alpha)(2 t-1)}{2+2 \gamma} h\right)+2 g^{\prime}(d) h(1-2 d) \leq 0$. 
Using the functional form for $g(d)$, this is equivalent to

$$
-(1-\beta)\left(1-F\left(-d+d^{2}+r-r^{2}\right)-\frac{(1+\alpha)(2 t-1)}{2+2 \gamma} h\right)-4(1 / 2-d)^{2} h \leq 0 .
$$

A sufficient condition for this inequality is

$$
-\left(1-F\left(-d+d^{2}+r-r^{2}\right)-\frac{(1+\alpha)(2 t-1)}{2+2 \gamma} h\right)-4(1 / 2-d)^{2} h \leq 0,
$$

or using the functional form for $F$,

$$
-\left(1 / 2-h\left(-d+d^{2}+r-r^{2}\right)-\frac{(1+\alpha)(2 t-1)}{2+2 \gamma} h\right) \leq 4(1 / 2-d)^{2} h .
$$

Rearranging terms, this inequality is equivalent to

$$
4(1 / 2-d)^{2}+\left(d-d^{2}-r+r^{2}\right) \geq \frac{(1+\alpha)(2 t-1)}{2+2 \gamma}-\frac{1}{2 h}
$$

or equivalently,

$$
1-3 d+3 d^{2}-r+r^{2} \geq-(1 / h)\left(\frac{1}{2}-\frac{(1+\alpha)(2 t-1)}{2+2 \gamma} h\right) .
$$

The left-hand side of this inequality is nonnegative for any $d \in[0,1 / 2]$, while the right-hand side is negative due to assumption 2.1 .

Lemma 7.4. If $F$ is uniform, in equilibrium, $0<\min \{d, r\}<1 / 2$.

Proof. Using lemma 7.2, we have that $\min \{d, r\} \geq 1 / 2$ implies $\min \{d, r\}>$ $1 / 2$.

Suppose $d=r>1 / 2$. Using assumption 2.1 and lemma 7.1, we can see that the derivative of the objective function of either party as given by equation 7.4 is negative, a contradiction.

Suppose $d>r>1 / 2$. Using the first order condition for either party, we obtain

$$
\begin{aligned}
g(d)+ & g(r)+G= \\
& -g^{\prime}\left(p_{i}\right)\left(1-F_{i}\left(-d+d^{2}+r-r^{2}\right)-\frac{(1+\alpha)(2 t-1)}{2+2 \gamma} h\right) / h\left(1-2 p_{i}\right)
\end{aligned}
$$

for $i=\mathbf{D}, \mathbf{R}$. Note that the left-hand side is independent of $i$. Thus,

$$
\begin{aligned}
-g^{\prime}(d)(1- & \left.F\left(-d+d^{2}+r-r^{2}\right)-\frac{(1+\alpha)(2 t-1)}{2+2 \gamma} h\right) / h(1-2 d)= \\
- & g^{\prime}(r)\left(F\left(-d+d^{2}+r-r^{2}\right)-\frac{(1+\alpha)(2 t-1)}{2+2 \gamma} h\right) / h(1-2 r),
\end{aligned}
$$


or equivalently,

$$
\frac{1-F\left(-d+d^{2}+r-r^{2}\right)-\frac{(1+\alpha)(2 t-1)}{2+2 \gamma} h}{F\left(-d+d^{2}+r-r^{2}\right)-\frac{(1+\alpha)(2 t-1)}{2+2 \gamma} h}=\frac{-g^{\prime}(r)(1-2 d)}{-g^{\prime}(d)(1-2 r)} .
$$

Using the functional form for $g$, we obtain,

$$
\frac{1-F\left(-d+d^{2}+r-r^{2}\right)-\frac{(1+\alpha)(2 t-1)}{2+2 \gamma} h}{F\left(-d+d^{2}+r-r^{2}\right)-\frac{(1+\alpha)(2 t-1)}{2+2 \gamma} h}=\left(\frac{d-1 / 2}{r-1 / 2}\right)^{2-\beta} .
$$

Since $d>r$ and $d+r>1$, we have $-d+d^{2}+r-r^{2}>0$, which implies $F\left(-d+d^{2}+r-r^{2}\right)>1 / 2$. Thus, the left-hand side is smaller than one. However, $d>r$ implies that the right-hand side is larger than one, a contradiction.

The case $r>d>1 / 2$ can be dealt with similarly.

Lemma 7.5. If $F$ is uniform, in equilibrium, $d=r<1 / 2$.

Proof. From the first order conditions, using (7.4),

$$
\begin{aligned}
g(d)+g(r)+ & = \\
& -g^{\prime}\left(p_{i}\right)\left(1-F_{i}\left(-d+d^{2}+r-r^{2}\right)-\frac{1+\alpha}{2+2 \gamma} h\right) / h\left(1-2 p_{i}\right)
\end{aligned}
$$

for $i=\mathbf{D}, \mathbf{R}$. Note that the left-hand side is independent of $i$. Thus,

$$
\begin{aligned}
-g^{\prime}(d)(1-F(-d & \left.\left.+d^{2}+r-r^{2}\right)-\frac{1+\alpha}{2+2 \gamma} h\right) / h(1-2 d)= \\
& -g^{\prime}(r)\left(F\left(-d+d^{2}+r-r^{2}\right)-\frac{1+\alpha}{2+2 \gamma} h\right) / h(1-2 r),
\end{aligned}
$$

or equivalently,

$$
\frac{1-F\left(-d+d^{2}+r-r^{2}\right)-\frac{1+\alpha}{2+2 \gamma} h}{F\left(-d+d^{2}+r-r^{2}\right)-\frac{1+\alpha}{2+2 \gamma} h}=\frac{-g^{\prime}(r)(1-2 d)}{-g^{\prime}(d)(1-2 r)} .
$$

Using the previous lemma, we only need to consider asymmetric equilibria satisfying $d<r<1 / 2$ or $r<d<1 / 2$. Consider the first case (the second case is similar). Then $-d+d^{2}+r-r^{2}>0$, which implies $F(-d+$ $\left.d^{2}+r-r^{2}\right)>1 / 2$. Thus, the left-hand side is smaller than one. However, if $d<r$, then the right-hand side is larger than one, a contradiction.

Theorem. 4.1 If $F$ is uniform on $[-\alpha, \alpha]$, there is a unique equilibrium, it is symmetric, and each party chooses the platform given implicitly by

$$
g^{\prime}\left(p^{*}\right)\left(\frac{1}{2}-\frac{1+\alpha}{2+2 \gamma}(2 t-1) h\right)+\left(2 g\left(p^{*}\right)+G\right) h\left(1-2 p^{*}\right)=0 .
$$

The solution to this equation has $0<p^{*}<1 / 2$. 
Proof. Since $F$ is assumed to be uniform on $[-\alpha, \alpha]$, we have

$$
f\left(-d+d^{2}+r-r^{2}\right)=h
$$

for any $d, r$.

Lemmas 7.1 to 7.5 imply that in equilibrium $d=r=p^{*}<1 / 2$, where (using equation 7.4 in the Appendix) $p^{*}$ satisfies the first order condition

$$
g^{\prime}\left(p^{*}\right)\left(\frac{1}{2}-\frac{1+\alpha}{2+2 \gamma}(2 t-1) h\right)+\left(2 g\left(p^{*}\right)+G\right) h\left(1-2 p^{*}\right)=0,
$$

which is equation 7.5 above.

To show that equation 7.5 has indeed a unique solution $p^{*} \in(0,1 / 2)$, note that $(2 g(p)+G) h(1-2 p)$ is continuous and strictly decreasing in $p$ for $p \in(0,1 / 2)$, since $g(p)>0$ and $g^{\prime}(p)<0$. Moreover, it takes the value $(2 g(0)+G) h$ for $p=0$ and the value 0 for $p=1 / 2$. The expression

$$
g^{\prime}(p)\left(\frac{1}{2}-\frac{1+\alpha}{2+2 \gamma}(2 t-1) h\right),
$$

is continuous and strictly decreasing in $p$ for $p \in(0,1 / 2)$, since $g^{\prime}(p)<0$, $g^{\prime \prime}(p)<0$, and (by assumption 2.1) $h(2 t-1)<(1+\gamma) /(1+\alpha)$. Moreover, it takes the value $g^{\prime}(0)(1 / 2-(1+\alpha) h(2 t-1) /(2+2 \gamma))$ for $p=0$ and decreases without bound as $p$ approaches $1 / 2$ from the left, since $g^{\prime}(p)$ decreases without bound as $p$ approaches $1 / 2$ from the left. Thus, a solution $p^{*} \in(0,1 / 2)$ to equation 7.5 exists if and only if

$$
(2 g(0)+G) h+g^{\prime}(0)(1 / 2-(1+\alpha) h(2 t-1) /(2+2 \gamma))>0,
$$

or equivalently

$$
h>\left(\frac{2(2 g(0)+G)}{-g^{\prime}(0)}+\frac{1+\alpha}{1+\gamma}(2 t-1)\right)^{-1} .
$$

which is implied by our parameter assumption 2.1. On account of the monotonicity, the solution must be unique.

Theorem. 5.1 If $F$ is uniform on $[-\alpha, \alpha]$, the equilibrium policy position $p^{*}$ is independent of $\delta$, increasing in $G, t$ and decreasing in $\alpha, \gamma$. Equilibrium campaign spending $E^{*}$ decreases with $\delta$ and increases with $G$. Moreover, if $G=0$,

$$
\frac{\partial E^{*}}{\partial t} \gtreqless 0 \Longleftrightarrow t \lesseqgtr 1 / 2+\frac{2 \alpha(1+\gamma)}{(2+\beta)(1+\alpha)}
$$

and

$$
\frac{\partial E^{*}}{\partial \alpha} \gtreqless 0 \Longleftrightarrow \alpha^{2}-(2 / \beta-1) \alpha+\frac{2 / \beta}{\frac{1+\gamma}{t-1 / 2}-1} \gtreqless 0 .
$$


Proof. Define

$$
H(p, \alpha, \gamma, t, G)=g^{\prime}(p)\left(\frac{1}{2}-\frac{1+\alpha}{2+2 \gamma}(2 t-1) h\right)+(2 g(p)+G) h(1-2 p) .
$$

From Theorem 4.1, $p^{*}$ is given by the solution to the equation

$$
H(p, \alpha, \gamma, t, G)=0
$$

under the constraint $0 \leq p \leq 1 / 2$. Moreover, $0<p^{*}<1 / 2$. It is simple to check that $\partial H / \partial p<0$ for $0<p<1 / 2$ (see the proof of Theorem 4.1). The comparative statics results for $p^{*}$ follow from taking derivatives of $H$ with respect to its arguments and employing the implicit function theorem.

With respect to campaign spending, from Theorem 3.2 and the definition of $c$ we get

$$
2 \delta(1+\gamma)\left(E^{*}\right)^{1+\gamma}=\frac{1+\alpha}{\alpha}(t-1 / 2)\left(2 g\left(p^{*}\right)+G\right) .
$$

Since $p^{*}$ is independent of $\delta$, an increase in $\delta$ can only be accommodated by a corresponding decrease in $E^{*}$.

Using Theorem 4.1, we can get that

$$
2 g\left(p^{*}\right)+G=\frac{\alpha \beta}{\left(1 / 2-p^{*}\right)^{2-\beta}}\left(1 / 2-\frac{1+\alpha}{2 \alpha(1+\gamma)}(t-1 / 2)\right) .
$$

Recall that $\partial p^{*} / \partial G>0$. Since the right-hand side of the expression above is increasing in $p^{*}$, it follows that $2 g\left(p^{*}\right)+G$ is increasing in $G$. Then, using equation 7.6, we get $\partial E^{*} / \partial G>0$.

If $G=0$, from equation 7.5 we obtain

$$
-g^{\prime}\left(p^{*}\right)\left(\frac{1}{2}-\frac{1+\alpha}{2+2 \gamma}(2 t-1) h\right)=2 g\left(p^{*}\right) h\left(1-2 p^{*}\right) .
$$

Using the functional form for $g$,

$$
\beta\left(1 / 2-p^{*}\right)^{\beta-1}\left(\frac{1}{2}-\frac{1+\alpha}{2+2 \gamma}(2 t-1) h\right)=2\left(1 / 2-p^{*}\right)^{\beta} h\left(1-2 p^{*}\right) .
$$

Simplifying,

$$
1 / 2-p^{*}=\left(\frac{\alpha \beta}{4}-\frac{1+\alpha}{4(1+\gamma)}(t-1 / 2) \beta\right)^{1 / 2} .
$$

Substituting in equation 7.6,

$$
\begin{aligned}
\delta(1+\gamma)\left(E^{*}\right)^{1+\gamma} & = \\
& \frac{(1+\alpha)(t-1 / 2)}{\alpha}\left(\frac{\alpha \beta}{4}-\frac{(1+\alpha)(t-1 / 2)}{4(1+\gamma)} \beta\right)^{\beta / 2}
\end{aligned}
$$


Thus the sign of $\partial E^{*} / \partial t$ is equal to the sign of the derivative with respect to $t$ of the expression in the right-hand side of equation 7.7. This derivative is proportional to

$$
\alpha \beta-\frac{(1+\alpha)(t-1 / 2)}{1+\gamma}(1+\beta / 2) .
$$

This expression is positive if

$$
t<1 / 2+\frac{2 \alpha(1+\gamma)}{(2+\beta)(1+\alpha)}
$$

Thus, if targeting accuracy is very small ( $t$ close to $1 / 2), \partial E^{*} / \partial t>0$. However, if targeting accuracy is large ( $t$ close to 1$)$, it is possible that a further increase in accuracy leads to a reduction in campaign spending.

As in the previous argument, the sign of $\partial E^{*} / \partial \alpha$ is equal to the sign of the derivative with respect to $\alpha$ of the expression in the right-hand side of equation 7.7. This derivative is proportional to

$$
-\frac{1}{\alpha^{2}}\left(\alpha-\frac{(1+\alpha)(t-1 / 2)}{1+\gamma}\right)+\frac{1+\alpha}{\alpha}\left(1-\frac{t-1 / 2}{1+\gamma}\right) \frac{\beta}{2} .
$$

This expression is proportional to the one in the statement of the Theorem. 


\section{REFERENCES}

[1] Calvert, R. (1985) "Robustness of the Multidimensional Voting Model: Platform Motivations, Uncertainty, and Convergence," American Journal of Political Science, 29, 69-95.

[2] Committee for the Study of the American Electorate (2005), "Turnout Exceeds Optimistic Predictions: More than 122 Million Vote," http://election04.ssrc.org/research/ csae 2004 final report.pdf

[3] Downs, A. (1957) An Economic Theory of Democracy, New York: Harper\&Row.

[4] Dekel, E., Jackson, M. and Wolinsky, A. (2004) "Vote Buying," mimeo.

[5] Feddersen, T. (2004) "Rational Choice Theory and the Paradox of Not Voting" Journal of Economic Perspectives, 18, 99-112.

[6] Jacobson, G. (2005) "Polarized Politics and the 2004 Congressional and Presidential Elections," Political Science Quarterly, 120, 199-218.

[7] Ledyard, J. (1984) "The Pure Theory of Large Two-Candidate Elections," Public Choice, 44, 7-41.

[8] Lindbeck, A. and Weibull, J. (1987), "Balanced-budget redistribution as the outcome of political competition,” Public Choice, 52, 273-297.

[9] Lindbeck, A. and Weibull, J. (1993), "A model of political equilibrium in representative democracy," Journal of Public Economics, 51, 195-209.

[10] McCarty, N., Poole, K. and Rosenthal, H. (2003) "Political Polarization and Income Inequality," mimeo.

[11] Morton, R. (1987) “A Group Majority Model of Voting," Social Choice and Welfare, 4, 17-31.

[12] National Election Studies, The NES Guide to Public Opinion and Electoral Behavior, http://www.umich.edu/ nes/nesguide/gd-index.htm

[13] Palfrey, T. and Rosenthal, H. (1983) "A Strategic Calculus of Voting," Public Choice, 41, 7-53.

[14] Palfrey, T. and Rosenthal, H. (1985) "Vote Participation and Strategic Uncertainty," American Political Science Review, 79, 62-78.

[15] Poole, K. and Rosenthal, H. (1997) Congress: A Political Economic History of Roll Call Voting, New York: Oxford University Press.

[16] Shachar, R. and Nalebuff, B. (1999) "Follow the Leader: Theory and Evidence on Political Participation," American Economic Review, 89, 525-547.

[17] Tilove, J. "Cutting-Edge Mobilization May Have Won the Day for Bush," http://www.newhousenews.com/archive/tilove/112604.html

[18] Wittman, D. (1983) "Platform Motivation: A Synthesis of Alternative Theories," American Political Science Review, 77, 142-157. 